\title{
Das Siegel des Išme-ilu, Eunuch des Nergal-ēreš, aus Dūr-Katlimmu
}

\author{
von Hartmut Kühne - Berlin und Karen Radner - London
}

Im Verlauf der Ausgrabungskampagne 2003 in Tall Šēh Hamad konnte in dem Grabungsabschnitt Mittlere Unterstadt II, Grabungsstelle „Neuassyrische Residenzen“, ein bemerkenswertes Objekt in stratifiziertem Kontext geborgen werden. Es handelt sich um das Fragment eines Gefäßdeckels (Abb. 1) ${ }^{1}$, dessen Oberfläche ursprünglich vollständig mit einem geglätteten Überzug versehen war, der aber größtenteils abgeblättert ist. Glücklicherweise ist das entscheidende, weil gesiegelte Fragment dieser Oberfläche am Objekt selbst erhalten geblieben (Abb. 2); in Ergänzung dazu konnten zum Teil winzige abgeplatzte und gesiegelte Fragmente gesammelt und zu insgesamt sechs relevanten Bruchstücken zusammengesetzt werden (Abb. 4-6). Daraus ergibt sich die Umzeichnung der Siegelabrollung (Abb. 7). Dieser Aufsatz mag das Musterbeispiel abgeben, das ich in meinen „Gedanken zur kunstgeschichtlichen Betrachtungsweise“ ${ }^{2}$ schuldig geblieben war.

Dem Direktor des Museums von Deir az-Zor, Yassir Shohan, und dem Kurator des Museums, Moin Ali, sei für die ständige Bereitschaft gedankt, das Objekt zugänglich zu halten. Marina Pucci war Leiterin der Grabungsstelle „Neuassyrische Residenzen“ und bearbeitet die „Stratigraphie und Architektur der Neuassyrischen Residenzen“ für die Reihe Berichte der Ausgrabungen Tall Šêh Hamad; ihr sei für den Plan Abb. 8 gedankt. Anja Fügert, die „Die neuassyrische Glyptik von Tall Šēh Hamad/Dūr-Katlimmu“ im Rahmen einer Dissertation bearbeitet, sei gedankt für die anregenden Diskussionen, die die Zeichnungen Abb. 1 bis 7 begleiteten, sowie einige wertvolle Hinweise. Hartmut von Wieckowski (Halle) hat das Wunder vollbracht, von diesen zerbrechlichen

1 Grabungsinventarnummer: SH 03/5953/0208. Das Objekt wird im Museum von Deir az-Zōr aufbewahrt, hat dort aber noch keine Museumsinventarnummer erhalten; die Fundjournalnummer des Museums (eine Art Vor-Registrierung) ist 03/0217.

2 H. Kühne, Gedanken zur kunstgeschichtlichen Betrachtungsweise, in: H. Kühne [u.a.] (Hg.), Fluchtpunkt Uruk. Archäologische Einheit aus methodischer Vielfalt. Schriften für Hans Jörg Nissen. Studia honoraria 6 (Rahden 1999) 342-351. 


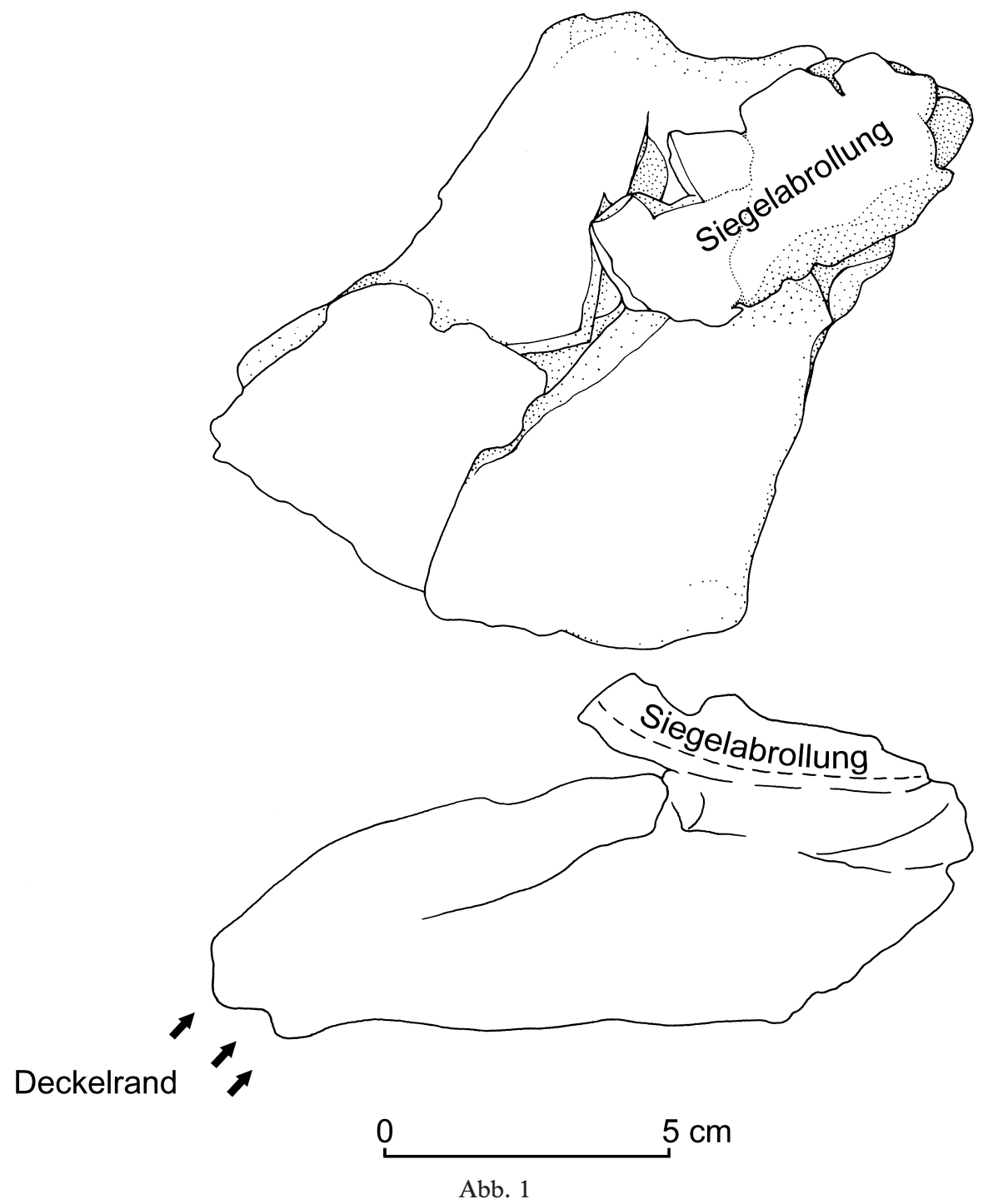


Fragmenten Abgüsse herzustellen. Konrad Wita sind die Aufnahmen von Abb. 2-6 zu verdanken.

\section{Die Siegelinschrift (Radner)}

Bei einem kurzen Aufenthalt in Berlin im Dezember 2003 las ich auf Bitte von Hartmut Kühne hin die Inschrift des Rollsiegels von den bei den Ausgrabungen hergestellten Abgüssen. Meine daraus resultierende Kopie wurde in die Umzeichnung der Siegelabrollung (Abb. 7) inkorporiert.

Die vierzeilige Inschrift ist im neuassyrischen Duktus geschrieben, wobei die verkürzte Zeichenform Lú* besonders typisch ist. Wie bei neuassyrischen Siegeln häufig festzustellen, ist die Inschrift auf dem Originalrollsiegel positiv geschnitten, weswegen sie in der Abrollung im $\mathrm{Ne}-$ gativ abgedrückt ist. Die Inschrift lautet:

(1) $\mathrm{NA}_{4} \cdot \mathrm{KIŠIB} \quad$,Siegel

(2) 'IŠ-mé-DINGIR des Išme-ilu,

(3) LÚ*.SAG $\check{s} a$ des Eunuchen des

(4) IdIGI.DU-KAM Nergal-ēreš.“

Mit Ausnahme der ungewöhnlichen Verwendung des Zeichens MI für den Lautwert /me/ (statt ME), was wohl durch das Bemühen um eine ausgewogene Zeichen- und Keilverteilung erklärt werden kann, die durch die Kürze des aus nur zwei Keilen bestehenden Zeichens ME aus dem Gleichgewicht gebracht worden wäre, bietet die Inschrift keinerlei sprachliche Besonderheiten, doch ist ihre Bedeutung für die Rekonstruktion der Geschichte von Dūr-Katlimmu außerordentlich wichtig: es handelt sich um das bislang älteste epigraphische Zeugnis, das aus der Unterstadt bekannt geworden ist (s. auch Abschnitt 3). Denn während ein Eunuch mit dem Allerweltsnamen Išme-ilu „Gott hat erhört“3 chronologisch nicht näher einzuordnen wäre, kann „Išme-ilu, der Eunuch des Nergal-ēreš,“ mit Sicherheit in das ausgehende 9. Jh. oder in das erste Viertel des 8. Jh. gestellt werden, die Wirkungszeit des bekannten Statthalters von Rașappa unter Adad-nērārī III. (810-783) und Salmanassar IV. (782-773).

3 Für weitere Träger dieses Namens in neuassyrischer Zeit s. R. Pruszinzsky, in: H. D. Baker (Hg.), The Prosopography of the Neo-Assyrian Empire 2/I: H-K (Helsinki 2000) 585 s.v. 


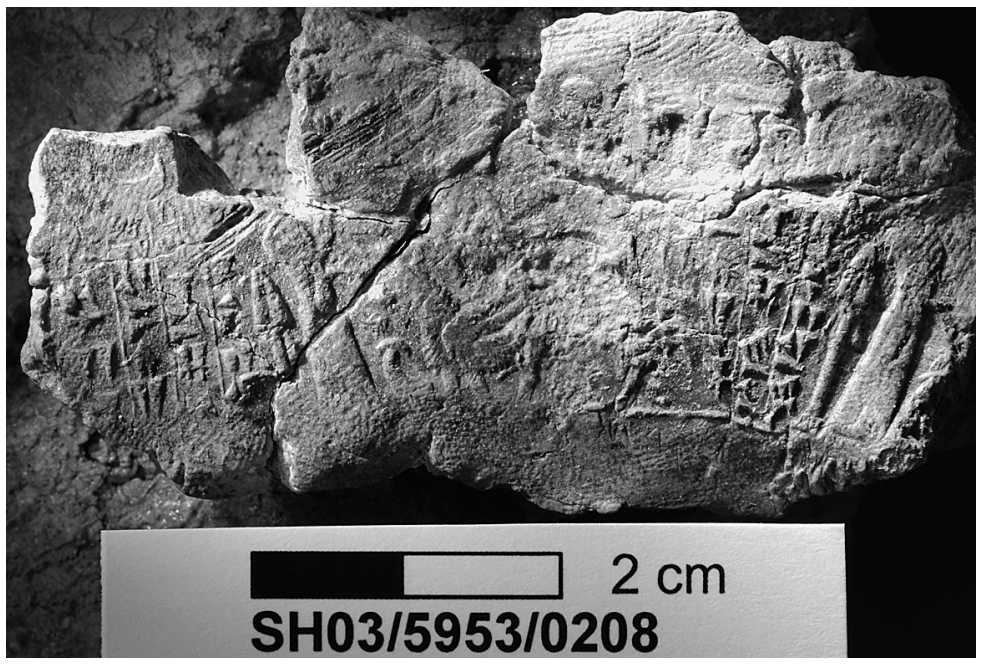

Abb. 2

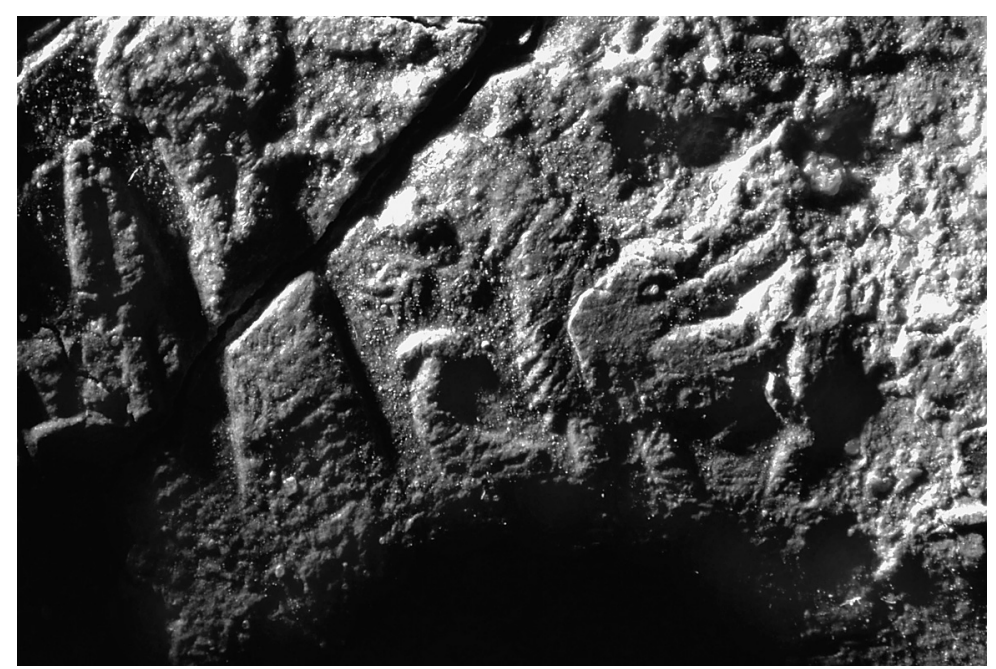

Abb. 3 


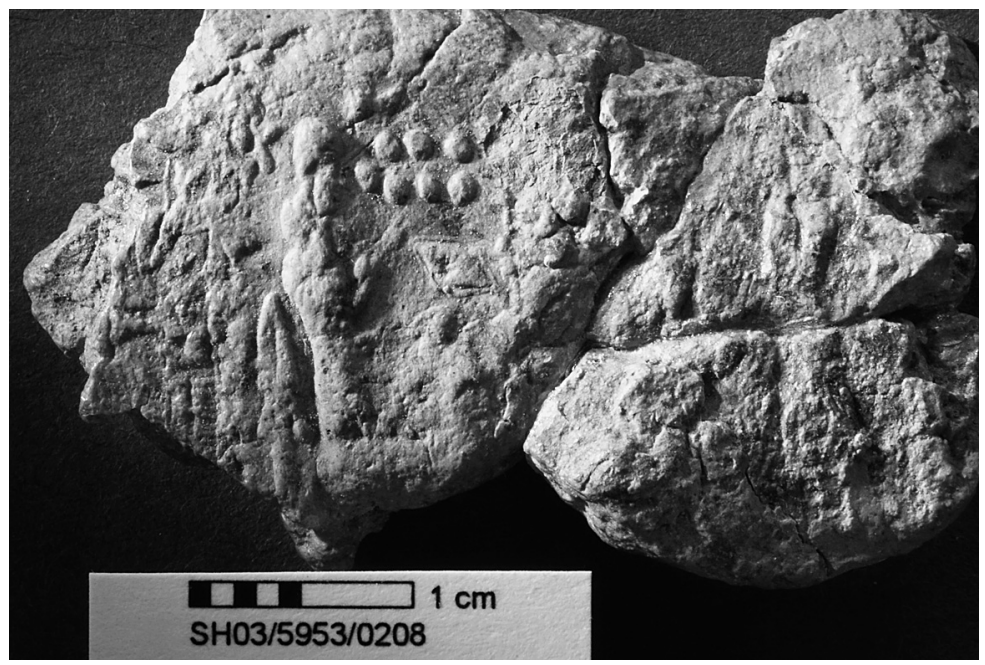

Abb. 4

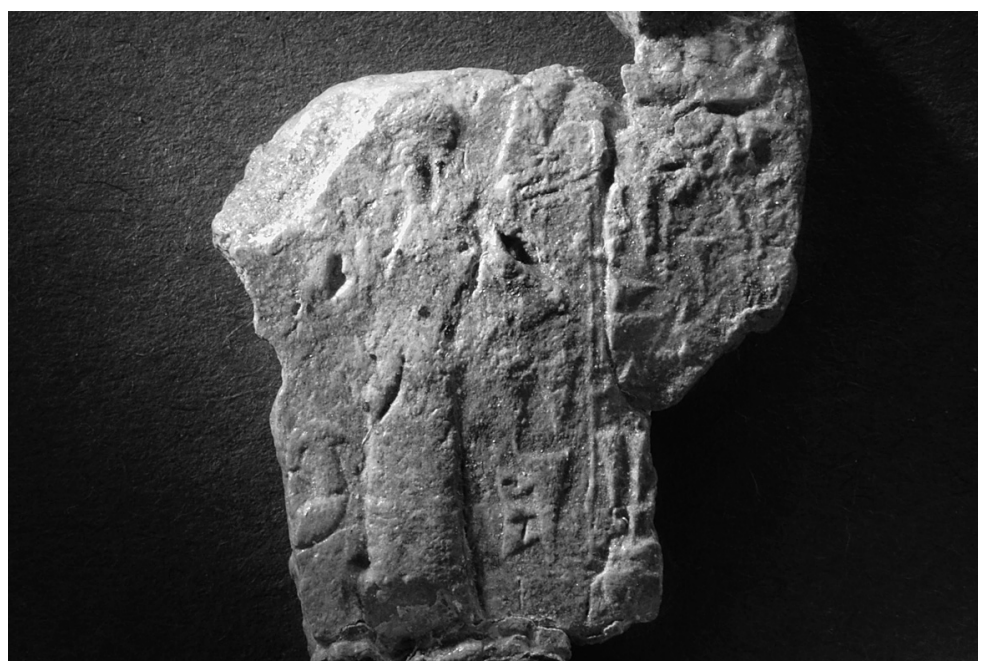

Abb. 5 


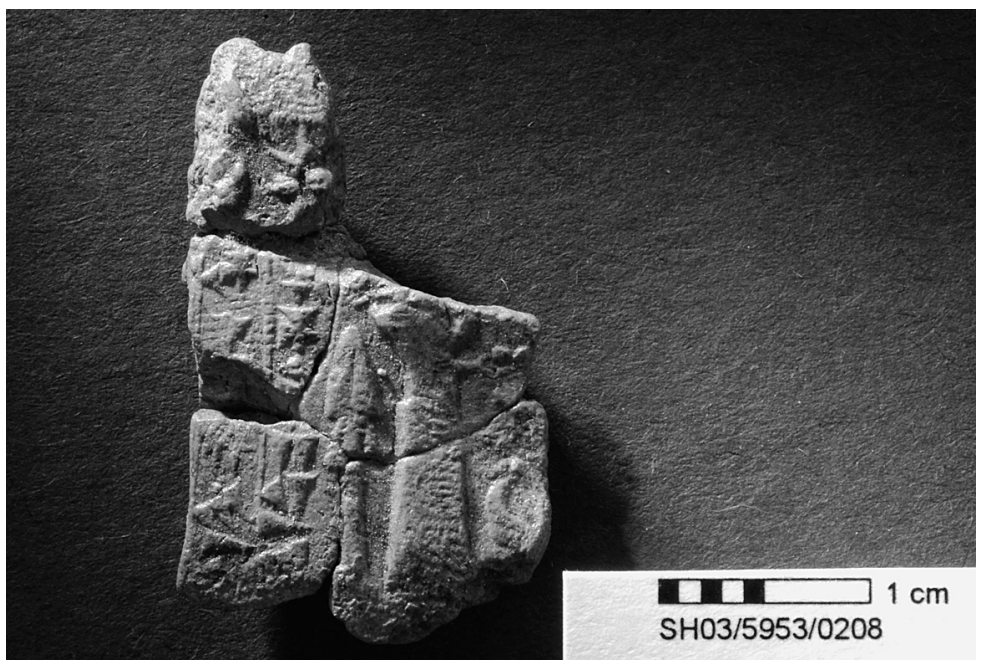

Abb. 6

A. Die Realisierung des Gottesnamens dIGI.DU im Neuassyrischen

Wie in allen anderen bereits bekannten Belegen für den Statthalter von Rașappa ist sein Name auch hier mit dem theophoren Element ${ }^{\mathrm{d}}$ IGI.DU geschrieben ${ }^{4}$, dessen Realisierung im Neuassyrischen sich bislang nicht zweifelsfrei entscheiden lässt. Dass gerade der Name des in zahlreichen und von unterschiedlichen Schreibern verfassten Texten be-

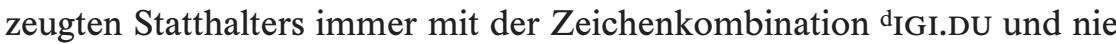
mit der sonst weitverbreiteten Zeichenkombination ${ }^{\mathrm{d}}$ MAŠ.MAŠ $=$ Nergal geschrieben wird, ist neben der Tatsache, dass beide Götternamen in denselben Texten nebeneinander belegt sind, ein schlagkräftiges Argument gegen die Gleichsetzung mit diesem Gott in neuassyrischen Texten ${ }^{5}$; eine Gleichsetzung, die allerdings für das Babylonische durchaus als gegeben angesehen werden kann ${ }^{6}$. Von einer Realisierung als Pālil im Neuassyrischen ist in Ermangelung von ausreichenden Indizien ebenfalls Abstand zu nehmen. Unser Siegel mag vielleicht einen Hinweis darauf liefern, dass die Sternliste V R 46: 7 mit der Gleichsetzung von digI.DU mit

${ }^{4}$ Für die übrigen Belege s. K. Åkerman/H. D. Baker, in: H. D. Baker (Hg.), The Prosopography of the Neo-Assyrian Empire 3/I: P-S (Helsinki 2002) 981-982 s.v. Pālilēreš.

5 So auch K. Åkerman/H. D. Baker, a.a.O. 981 (mit früherer Literatur) und M. Krebernik, dPALIL(IGI.DU), R1A 10 (2003-2005) 281.

${ }^{6}$ Dazu Krebernik, a.a.O. 


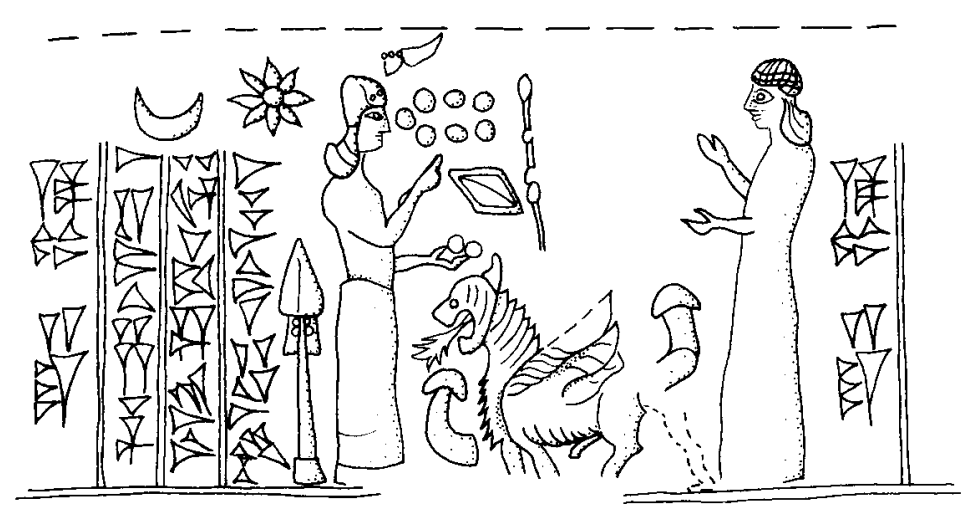

Abb. 7

Alammuš, dem Wesir des Mondgottes ${ }^{7}$, ernstzunehmen ist. Falls es gestattet ist, eine Beziehung zwischen dem theophoren Element im Namen des Statthalters und der auf dem Siegel seines Eunuchen dargestellten Gottheit anzunehmen (und das ist beileibe nicht sicher!), dann ist vielleicht die Beobachtung wichtig, dass der Gott auf seinem Löwendrachen in einem Boot zu stehen scheint, wie es sonst dem Mondgott vorbehalten ist.

Wir halten hier also wieder einmal fest, dass die Realisierung des Namens des Statthalters von Rașappa unter Adad-nērārī III. und Salmanassar IV. nicht bekannt ist und dass beide in der Sekundärliteratur gebräuchliche Lesungen als Nergal-ēreš oder als Pālil-ēreš unwahrscheinlich sind. Im Folgenden verwenden wir aber aus praktischen Gründen und im Wissen, dass dies sicherlich nicht der Aussprache im Neuassyrischen entspricht, die heute weithin gebräuchliche Realisierung Nergal-ēreš.

\section{B. Nergal-ēreš und Dūr-Katlimmu}

Nergal-ēreš' Verbindung mit Dūr-Katlimmu war schon vor der Auffindung der Abrollung des Siegels seines Untergebenen Išme-ilu bekannt, denn seine Domäne umfasste nach der Aufzählung der Königsstele von Saba'a auch diese $\mathrm{Stadt}^{8}$; terminus post quem für die Abfassung

7 Die „Kleinen Zwillinge“ Alammuš und Ningublaga werden hier als digI.DU und Ningublaga erklärt; beachte, dass Krebernik, a.a.O. hier eine Kontamination mit den „Großen Zwillingen“ Lugalerra und Meslamta'ea (= Nergal) für möglich hält.

8 A. K. Grayson, Assyrian Rulers of the Early First Millennium BC II (858-745 BC). Royal Inscriptions of Mesopotamia: Assyrian Periods 3 (Toronto 1996) A.0.104.6: 24 [= RIMA 3]; J. Börker-Klähn, Altvorderasiatische Bildstelen und vergleichbare Felsreliefs. Baghdader Forschungen 4 (Mainz 1982) Nr. 163. 
dieser Inschrift ist das Jahr 797, als Adad-nērārī III. den Landstrich Hindānu zu den von Nergal-ēreš kontrollierten Ländern hinzufügte ${ }^{9}$.

Dass Nergal-êreš nun durch das Siegel des Išme-ilu erneut mit der Stadt Dūr-Katlimmu in engste Beziehung gebracht wird, sichert die $\mathrm{Zu}$ weisung einer fragmentarischen Königsstele des Adad-nērārī III. an diesen Beamten weiter $\mathrm{ab}^{10}$, der sich in einer zweiten, an der linken Seite der Stele angebrachten Inschrift als der Urheber dieses Königsbildes ausweist und dessen Name später unleserlich gemacht wurde. Von dieser Stele sind bisher zwei Bruchstücke bekannt geworden. Das erste Fragment wurde von Hormuzd Rassam im Jahr 1879 in Tall Šēh Hamad gefunden und befindet sich heute im British Museum ${ }^{11}$. Ein zweites Bruchstück wurde im Jahr 2000 bei Christie's New York angeboten ${ }^{12}$, fand aber damals, wie mir John M. Russell 2001 mitteilte, keinen Käufer; zu seinem derzeitigen Verbleib ist mir nichts bekannt, doch mag es sich noch in derselben europäischen Privatsammlung befinden, der es angeblich seit den 1960ern angehörte. Die Stele zeigt das Abbild des nach rechts gewandten, betenden assyrischen Königs mit den Göttersymbolen und ist sowohl auf der Vorderseite wie auch auf der linken Seite der Stele mit Keilinschriften versehen. Die 32-zeilige Inschrift auf der Vorderseite lässt sich anhand der beiden Fragmente nahezu vollständig rekonstruieren ${ }^{13}$ und berichtet von einem Feldzug Adad-nērārīs III. (811-783) gegen das Hethiterland, also Nordsyrien, und speziell gegen Atar-šumkī von Arpad, der in das Jahr 805 datiert werden kann ${ }^{14}$; weiters ist die Rede von Bauarbeiten am Tempel des Gottes Salmānu, den „mein Vater Sal-

9 RIMA 3, A.0.104.9 = L. Kataja/R. Whiting, Grants, Decrees and Gifts of the Neo-Assyrian Period. State Archives of Assyria 12 (Helsinki 1995) Nr. 85.

10 Wie bereits von A. R. Millard/H. Tadmor, Adad-nirari III in Syria: Another stele fragment and the dates of his campaigns, Iraq 35 (1973) 60, Grayson, a.a.O. 206, und K. Radner, Die neuassyrischen Texte aus Tall Šêh Hamad. Beiträge zu den Ausgrabungen von Tall Šêh Hamad 6 (Berlin 2002) 15 (im Folgenden: Radner 2002) angenommen.

11 RIMA 3, A.0.104.5; Börker-Klähn, a.a.O. Nr. 165; Millard/Tadmor, a.a.O. Tf. 29.

12 Das Stelenbruchstück wurde am 13. Juni 2000 von Christie's New York bei einer Antiquities Auction als lot 491 zum Verkauf angeboten; gute Abbildungen im Katalog, wo das Stück fehlerhaft als „A Neo-Assyrian Stele of Shamshi-Adad V“ geführt wird: die falsche Zuschreibung beruht auf der Fehlinterpretation der Passage „Salmanassar, mein Vater", was als Hinweis auf diesen Sohn Salmanassars III. gewertet wurde; gemeint ist aber der Vorfahr Salmanassar I. S. dazu Radner 2002 (Anm. 10) 15.

13 Von den 32 Zeilen der Inschrift sind die ersten zehn Zeilen auf dem Fragment im British Museum und die folgenden 22 Zeilen auf dem bei Christie's angebotenen Fragment erhalten; Fehlstellen gibt es an den Zeilenanfängen des Bruchstücks aus dem British Museum und in der Bruchstelle zwischen den beiden Stücken.

14 Dazu s. Millard/Tadmor, a.a.O. 57-64. 
manassar“ - ${ }^{\mathrm{d}} \mathrm{DI}-m a-n u$-MAŠ $a-b i$, d.h. der Vorfahr Salmanassar I. (1263-123415), dem die Gründung dieses Tempels zugeschrieben werden kann ${ }^{16}$ - gebaut und den nun Adad-nērārī renoviert hatte. Von der Inschrift auf der linken Stelenseite sind die letzten 25 Zeilen erhalten. Sie ist nicht im Namen des Königs, sondern im Namen eines Beamten abgefasst, dessen Name zu einem späteren Zeitpunkt getilgt wurde, und beschreibt die von diesem am Heiligtum durchgeführten Arbeiten, unter anderem die Aufstellung eines Bildnisses seines Herrn Adad-nērārī, dessen Entfernung aus der Gegenwart des Gottes Salmānu durch Fluchklauseln verhütet werden soll: der Name dieses Beamten ist zweifellos als Nergal-ēreš zu rekonstruieren.

\section{Das Siegelbild (Kühne)}

\section{A. Vorikonographische Beschreibung}

Die Fundumstände (Abb. 8) des Gefäßdeckels (Abb. 1) sind so ungewöhnlich wie das Objekt selbst. Das Stück lag in der - von Osten (rechts) gezählt - zweiten von vier Mulden, die in den anstehenden Boden eingetieft sind. Der anstehende Boden ist deutlich sichtbar zu einer planen Fläche abgearbeitet und als Fußboden genutzt worden. Die Mulden waren folglich Installationen, die von diesem Fußboden aus angelegt worden waren. Diese Anlage gehört zur Erdphase 9 der Unterstadt II ${ }^{17}$. Mit dem Fußboden lassen sich jedoch keine Mauern in Verbindung bringen, sondern diese sind durch die Fundamentgruben der jüngeren Mauern der Erdphase 8 vollständig abgetragen worden. Die an dieser Stelle in Erdphase 8 bestehenden Räume V und R gehören zu Haus („Residenz“) 1; deren nur ca. 10 Zentimeter höher liegender Fußboden versiegelte den Befund der Erdphase 9. Die Mulden dürften als Halterungen für spitzbödige Gefäße zu interpretieren sein, die mit Gefäßdeckeln verschlossen waren. Unser Fragment und weitere in den Mulden angetroffene Objekte sind wahrscheinlich bei der Aufgabe der Räumlichkeiten dorthin gelangt und gehörten zu dem Rauminventar.

15 Nach dem Datierungsvorschlag von J. Boese/G. Wilhelm, Aššur-dan I., Ninurta-apilEkur und die mittelassyrische Chronologie, Wiener Zeitschrift für die Kunde des Morgenlandes 71 (1979) 19-38.

16 K. Radner, Der Gott Salmānu („Šulmānu“) und seine Beziehung zur Stadt Dūr-Katlimmu, Die Welt des Orients 29 (1998) 50.

17 H. Kühne, Tall Šaih Hamad (Archäologisch), R1A 11, im Druck. 


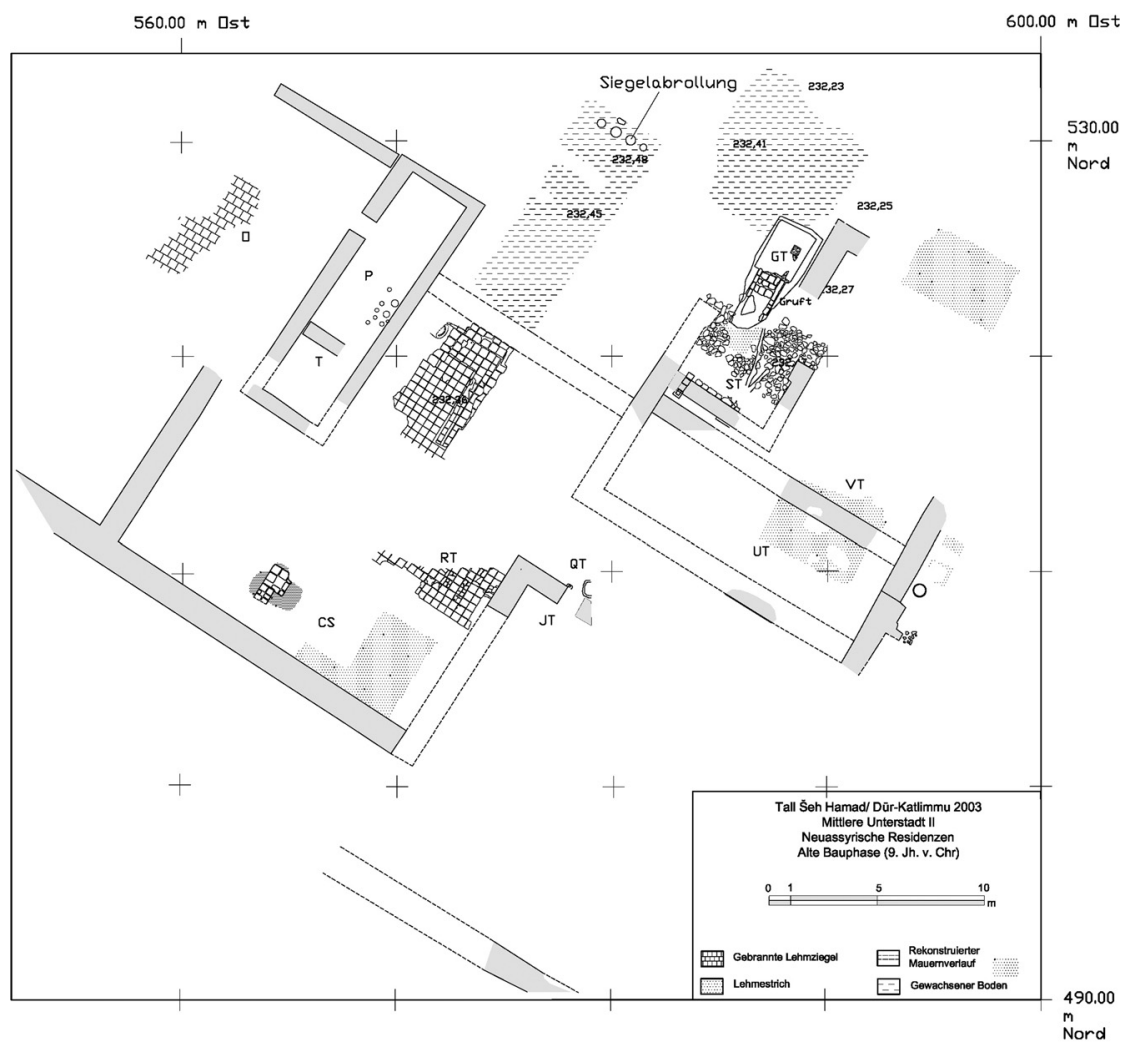

Abb. 8

Die Bildszene ist vollständig auf der erhaltenen Oberfläche des Gefäßdeckels (Abb. 2) abgerollt. Das Siegel hatte eine Höhe von 2,3 cm. Die untere Standlinie ist partiell erhalten (Abb. 2, 5), und eine obere Bildbegrenzung ist durch den Abdruck des Randes der Abrollung gegeben (Abb. 2, 4). Das Rollsiegel war wahrscheinlich gefasst: die linear aufgereihten Kügelchen am oberen Rand (Abb. 4) könnten so gedeutet werden. Links und rechts stehen sich je eine Person einander zugewandt gegenüber (Abb. 2-7). Die linke Person ist von Göttersymbolen umgeben (Abb. 4, 7), hinter ihr der Spaten des Marduk; links von ihrem Kopf, über der Inschrift, der Stern der Ištar und die Mondsichel des Sin; vor ihrem Gesicht das Siebengestirn der Sebetti, darüber ein nicht deutbarer, hornartiger Gegenstand und darunter das Symbol der Raute, neben der noch ein stabförmiger Gegenstand mit vier Verdickungen zu erkennen ist. 
Die rechte Person ist schemenhaft auf Abb. 4 und deutlicher auf Abb. $5 \mathrm{zu}$ erkennen. Beide Personen sind bartlos, tragen ein langes Schalgewand und haben eine ähnliche Armhaltung: den einen Unterarm gerade und den anderen schräg nach oben vorgestreckt. Die linke Person hält in ihrer linken Hand zwei kugelförmige Gegenstände (Abb. 4, 6), während der Zeigefinger der rechten Hand ausgestreckt ist. Dagegen hält die rechte Person beide Hände nach oben geöffnet (Abb. 7). Auch in den Kopfbedeckungen und in der Haaranordnung unterscheiden sie sich voneinander: die linke trägt eine kalottenförmige Kappe mit verziertem Rand, unter der das Haar hervorquillt und in einem dicken gestaffelten Bausch in den Nacken fällt (Abb. 4), während die rechte Person eine kalottenförmige Frisur mit einem einfachen Haarbausch im Nacken hat (Abb. 5). Die Physiognomie beider Personen ist ähnlich und gut im Detail erkennbar (Abb. 4, 5).

Im Feld zwischen den beiden Personen ist im unteren Teil ein aufrecht auf seinen vier Beinen stehender, feuerspeiender, geflügelter Löwendrache mit Rinderohr, aber ohne Stierhorn dargestellt (Abb. 2, 3), dessen rechter Hinterlauf schemenhaft auszumachen ist, dessen Füsse und Schwanz aber nirgends abgedrückt sind. Vor und hinter ihm ragt je ein gebogener Gegenstand mit einem pilzförmigen Aufsatz hervor (Abb. 4, 5): das vor der Brust des Löwendrachen befindliche Objekt ist offenbar vollständig wiedergegeben, bei dem hinter ihm befindlichen fehlt dagegen der vordere Abschluss. Der obere Teil des Feldes ist in den Abdrücken dermaßen undeutlich erhalten (Abb. 2-3), dass keine figürliche Darstellung mit Sicherheit ausgemacht werden kann. Am linken Rand dieses Bildfeldes, in Abgrenzung zu dem Siebengestirn und der Raute, befindet sich ein senkrecht angebrachter stabförmiger Gegenstand mit vier kugelförmigen Verdickungen. Die Inschrift beginnt hinter der rechten Person und endet am Spatensymbol; sie besteht aus vier Zeilen, die durch Linien voneinander abgesetzt sind (Abb. 2). Die Bilddarstellung des Siegels wurde sehr detailreich und plastisch eingraviert, was an dem Löwendrachen (Abb. 2) und an den beiden Personen (Abb. 3-5) nachvollzogen werden kann. 


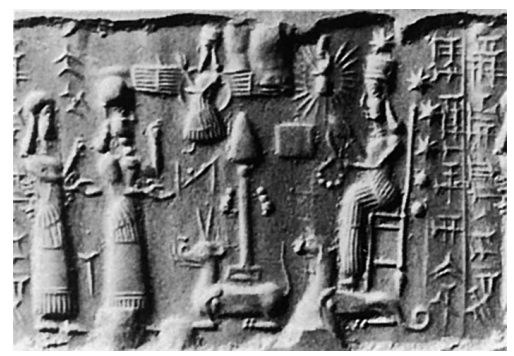

Abb. 9

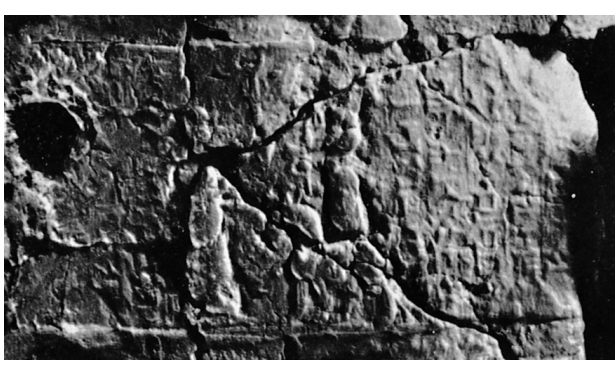

Abb. 10a

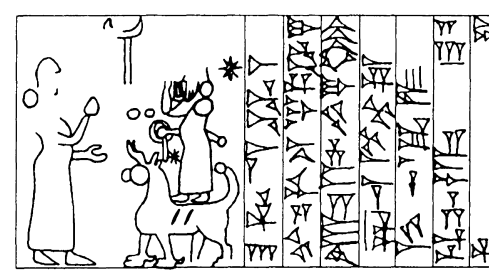

Abb. 10b

\section{B. Ikonographische Analyse}

Auf Grund seiner Inschrift reiht sich das Siegel in die Gruppe der Eunuchen-Siegel ein ${ }^{18}$. Eunuchen wirkten im Königreich Assyrien als hohe Staatsbeamte und lassen sich in dieser Funktion seit der mittelassyrischen Zeit belegen ${ }^{19}$. Auffallend ist eine Gruppe von insgesamt zehn Siegeln von Eunuchen und Statthaltern, die dem König Adad-nērārī III. unterstellt waren ${ }^{20}$ (Abb. 9-12), von dem auch ein eigenes Siegel überliefert ist $^{21}$. Darunter befindet sich auch das Siegel des Statthalters von Rașappa, Nergal-ēreš, ein reines Schriftsiegel ${ }^{22}$, und das Siegel des Pān-Aššur-lāmur, Statthalter von Aššur ${ }^{23}$ (Abb. 11). Mehrfach sind Siegel von

\footnotetext{
18 K. Watanabe, Neuassyrische Siegellegenden, Orient 29 (1993) 114-120 (im Folgenden: Watanabe 1993).

19 C. Fischer, Die Bildsymbolik der Assyrer in der akkadischen Tradition, Altorientalische Forschungen 27 (2000) 308-324.

20 Watanabe 1993 (Anm. 18) Nr. 5.1, 5.2, 6.1-6.6, 7.1, 9.2.

21 Watanabe 1993 (Anm. 18) Nr. 2.1.

22 Watanabe 1993 (Anm. 18) Nr. 5.1.

23 Watanabe 1993 (Anm. 18) Nr. 9.2.
} 


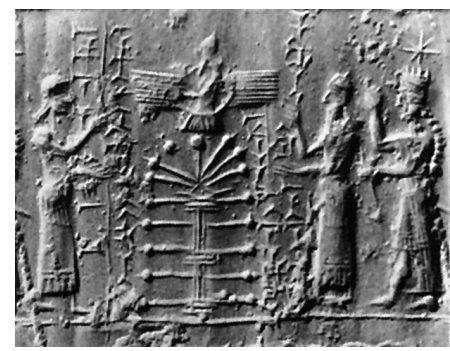

Abb. 11

Eunuchen bezeugt, die Statthaltern unterstanden, darunter das Siegel des Rēmanni-ilī, des Eunuchen des Bēl-tarși-ilumma, Statthalter von Kalhu und Eponym des Jahres 797 (Abb. 12)24; das Siegel des Bruders jenes Statthalters ist ebenfalls bekannt ${ }^{25}$. Neben dem hier vorgestellten Siegel des Išme-ilu ist noch ein weiteres Siegel eines Eunuchen des Statthalters Nergal-ēreš belegt, nämlich das Siegel des Aššur-bēlu-uṣur (Abb. 13) ${ }^{26}$. Alle Siegel zeichnen sich durch hervorragende Qualität, Plastizität, klare Bildanordnung und motivische Vielfalt aus, und diese Merkmale charakterisieren auch das Siegel des Išme-ilu.

Die beiden Personen auf seinem Siegel stehen einander isokephal gegenüber. Eine ähnliche Komposition der Personenanordnung ist auf dem Siegel des Pān-Aššur-lāmur, des Statthalters von Aššur (Abb. 11)27, anzutreffen. Der Unterschied zwischen den beiden Personen ist ikonographisch eindeutig und deshalb sicher zu deuten: rechts steht, von einer Gottheit flankiert, der König Adad-nērārī (III.) höchstpersönlich, erkennbar an seiner Königsmütze, während ihm gegenüber der Siegelinhaber Pān-Aššur-lāmur abgebildet ist, dessen Kappe und dicker Haarwulst im Nacken dem der linken Person auf dem Išme-ilu-Siegel ähneln. Die Frisur der rechten Person des Išme-ilu-Siegels - kalottenförmig mit einem einfachen Haarbausch - entspricht dagegen der des Eunuchen Aššur-bēlu-uṣur in dessen Siegel (Abb. 13). Der Unterschied in der Haltung, Ausstattung und Anordnung der beiden Personen auf dem Siegel des Išme-ilu erklärt sich höchstwahrscheinlich ebenfalls aus ihrem unterschiedlichen gesellschaftlichen Rang (s.u.).

\footnotetext{
24 Watanabe 1993 (Anm. 18) 116, Nr. 6.5.

25 Watanabe 1993 (Anm. 18) 118 Nr. 7.1.

26 Watanabe 1993 (Anm. 18) 114 Nr. 6.1.

27 Watanabe 1993 (Anm. 18) Nr. 9.2.
} 


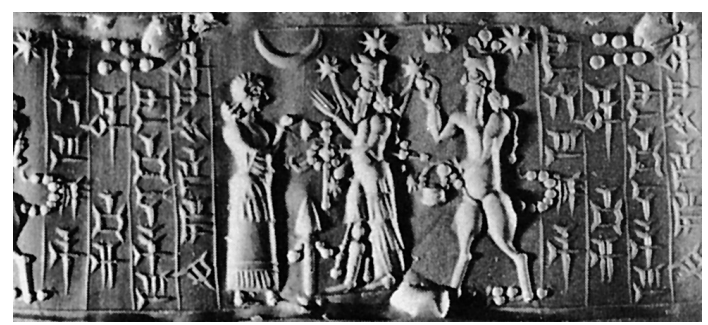

Abb. 12

In dem Raum zwischen den beiden Personen muss sich über dem Löwendrachen eine anthropomorphe Darstellung befunden haben. Der für sie vorhandene Raum ist knapp; diese anthropomorphe Abbildung wäre merklich kleiner als die beiden links und rechts von ihr stehenden Personen, hätte aber in der Breite genügend Platz zur Verfügung, weil die Figuren schlank geschnitten sind. Von den Proportionen vergleichbar ist die Anordnung der Figuren auf dem Siegel des Bēl-tarși-ilumma, des Statthalters von Kalhu (Abb. 10). Der mit Kugeln besetzte Stab muss sich in der linken Hand dieser Person befunden haben, wobei die nicht geschlossene Kugel die Hand markieren könnte (Abb. 7). Diese Person muss folglich eine Gottheit darstellen, denn der Zepter-ähnliche Stab ist ein Göttern vorbehaltenes Requisit. Aus der Gruppe der Adad-nērārī III.-zeitlichen Siegel kann dafür allerdings kein Beispiel angeführt werden; vergleichbar ist aber das Siegel des Sadibbu (Abb. 15)28, das dieser Gruppe nahesteht.

Der beste Vergleich zu dem Löwendrachen des Išme-ilu-Siegels findet sich auf einem Siegel aus der Bibliothèque Nationale in Paris (Abb. 16) ${ }^{29}$, das stilistisch sehr gut zu der Gruppe der Adad-nērārī III.-Siegel passt. Es bildet den Wettergott Adad ab, der eine Axt in der linken Hand hält und auf einem aufrecht auf vier Vogelfüßen stehenden, feuerspeienden, geflügelten und mit einem Skorpionschwanz ausgestatteten Löwendrachen steht ${ }^{30}$. Aus der hier betrachteten Gruppe der Adad-nērārī III.-zeitlichen Siegel kann nur das des Bēl-tarși-ilumma (Abb. 10) angeführt werden, auf dem der Löwe(?) allerdings flügellos ist. Dazu passt auch die Darstel-

28 B. Buchanan, Catalogue of Ancient Near-Eastern Seals in the Ashmolean Museum I (Oxford 1966) 114, Nr. 632.

29 L. Delaporte, Catalogue des cylindres orientaux et des cachets de la Bibliothèque Nationale (Paris 1910) pl. XXIV: 355.

30 E. A. Braun-Holzinger, Löwendrache, RlA 7 (1987-1990) 97-99, § 3 c. 


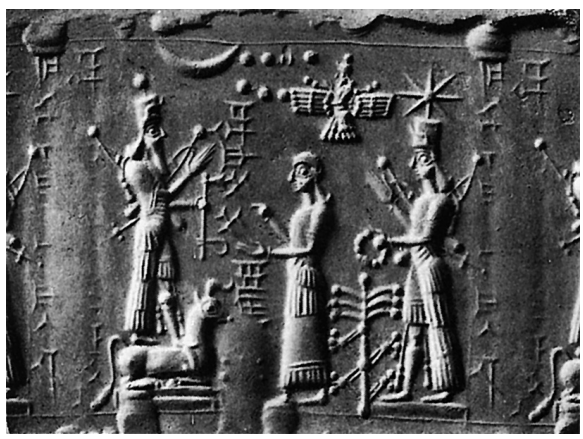

Abb. 13

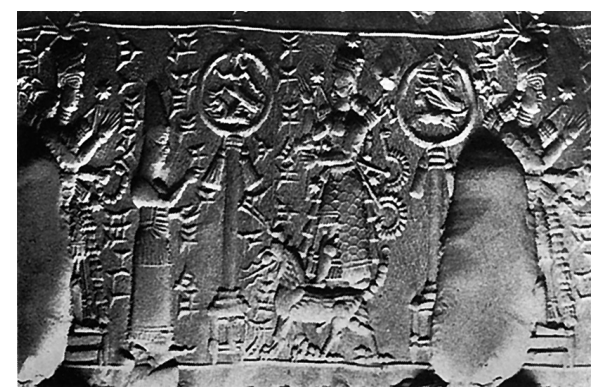

Abb. 14

lung eines Löwen auf dem Siegel des Aššur-šumu-iddina (Abb. 14) ${ }^{31}$, dessen Hinterbeine Vogelfüße sind.

Problematisch sind die beiden gebogenen Gegenstände mit pilzförmigem Aufsatz auf dem Siegel des Išme-ilu (Abb. 4, 5), deren unterschiedlich hohe Positionierung beabsichtigt zu sein scheint. Auf dem zeitlich wohl nicht allzu weit von der Adad-nērārī III.-zeitlichen Gruppe einzuordnenden Siegel des Handelsaufsehers Mīnu-ēpuš-ana-ili ${ }^{32}$ sind in ähnlich versetzter Anordnung zwei voluminöse T-förmige Gebilde vor und hinter den Beinen des Gottes angebracht. Bei den pilzförmigen Objekten auf dem Išme-ilu-Siegel könnte es sich um die Darstellung eines Bootes handeln. Auf dem bereits erwähnten Siegel des Sadibbu (Abb. 15) ist der Wettergott auf einem Boot stehend abgebildet, das mit entenkopfförmi-

31 Watanabe 1993 (Anm. 18) 112 Nr. 4.1.

32 Watanabe 1993 (Anm. 18) 118 Nr. 7.2. 


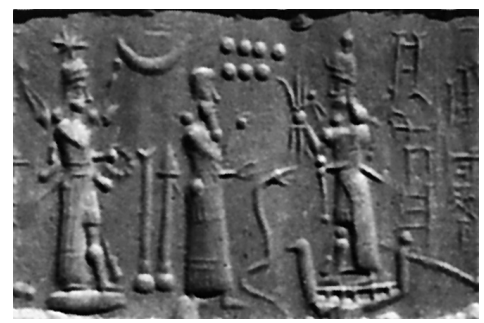

Abb. 15

gen Steven ausgestattet ist, von denen der hintere leicht höher als der vordere angeordnet ist. Normalerweise ist das Boot ein Requisit des Mondgottes $^{33}$, der z.B. auf einem von Felix Blocher als mittelassyrisch klassifiziertem Siegel in einem Boot steht ${ }^{34}$, dessen Steven nach innen eingerollt sind (Abb. 17) ${ }^{35}$. Obwohl nicht auszuschließen ist, dass es sich bei den pilzförmigen Objekten auf dem Išme-ilu-Siegel um die symbolische Wiedergabe eines Bootes handeln könnte, wird diese These dadurch geschwächt, dass mit Sicherheit kein vollständiges Boot dargestellt war, denn die pilzförmigen Objekte stehen jeweils abgeschlossen für sich (s. oben Abschnitt 2.A). Für die Annahme, dass das Siegel vielleicht nachgeschnitten wurde, liefern die Abdrücke keinerlei Hinweise.

\section{Ikonologie}

Beim Siegel des Pān-Aššur-lāmur, des Statthalters von Aššur unter Adad-nērārī III. (Abb. 11), sind die auf Augenhöhe sich gegenüber stehenden Personen - der Statthalter selbst und sein König - subtil, aber dennoch eindeutig durch ihre Kopfbedeckungen in ihrem hierarchischem Verhältnis zueinander gekennzeichnet. In Analogie lassen sich auch die beiden Personen auf dem Išme-ilu-Siegel mit großer Sicherheit deuten: rechts steht der Eunuch Išme-ilu, links sein Vorgesetzter Nergalēreš, seinerseits Eunuch des Königs Adad-nērārī III. Nergal-ēreš huldigt im sogenannten ubāna tarāṣu-Gestus seinem Gott (s. sogleich) und ist

33 E. A. Braun-Holzinger, Die Ikonographie des Mondgottes in der Glyptik des III. Jahrtausends v.Chr., Zeitschrift für Assyriologie 83 (1993) 119-135; H. Kühne, Der Gott in der Mondsichel, Altorientalische Forschungen 24 (1997) 375-382.

${ }^{34}$ F. Blocher, Nachlese zur Ikonographie des Mondgottes. Nabonids Stele aus Harran (H1B), in: R. M. Boehmer/J. Maran (Hg.), Lux orientis: Festschrift für Harald Hauptmann zum 65. Geburtstag (Rahden 2001) 45-48.

35 N. Özgüç, Samsat Mühürleri, Belleten 51 (1987) 436 Nr. 13. 


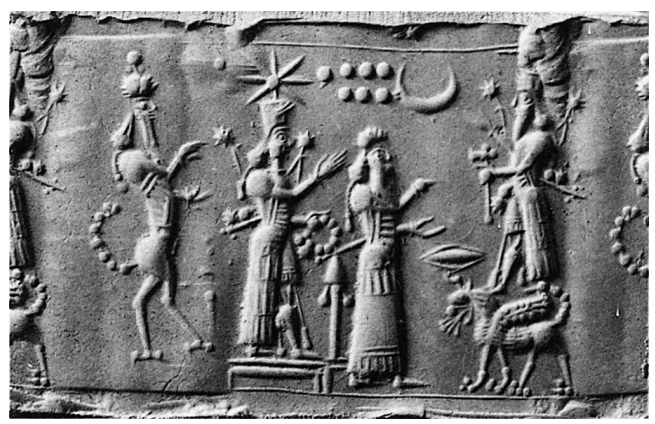

Abb. 16

von den Göttersymbolen umgeben, die durch die Bildanordnung auf ihn Bezug zu nehmen scheinen, was in dieser Form ungewöhnlich und nur dem assyrischen König vorbehalten ist: Nergal-ēreš ist dadurch auf dem Siegel seines Untergebenen in einen königsgleichen Stand erhoben. Dieser hier bildlich angedeutete Hochmut ist bekanntlich vor den Fall gekommen, insofern als die riesige Domäne des mächtigen und lang gedienten Nergal-ēreš, der zum Zeitpunkt seines zweiten Eponymats im Jahr 775 ja schon mindestens 28 Jahre das Amt des Statthalters von Rașappa ausgeübt hatte, später zerschlagen wurde ${ }^{36}$ und sein Name einer damnatio memoriae anheimfiel ${ }^{37}$.

Was bleibt, ist ein Versuch, die Identität des auf unserem Siegel dargestellten Gottes zu klären, der zwischen den beiden Personen auf seinem Löwendrachen steht, dem Nergal-ēreš zugewandt und mit einem Zepter in seiner linken Hand. Der Löwendrache und der Zepter-ähnliche Stab sind die einzigen Indizien, durch die der Gott bestimmt werden könnte. Wie bereits gezeigt wurde, findet sich das beste Vergleichsbeispiel für den Löwendrachen auf einem Siegel aus der Bibliothèque Nationale (Abb. 16), hier als Träger des Wettergottes Adad, der durch seine Axt identifiziert werden kann. Der Wettergott mit der Axt ist auch auf dem Siegel des Aššur-bēlu-ușur dargestellt, wie Išme-ilu ein Eunuch des Nergal-ēreš (Abb. 13), hier allerdings auf seinem angestammten Symboltier, dem Stier, während er auf dem Siegel des Eunuchen Rēmanni-ilī ohne Symboltier abgebildet ist (Abb. 12). Auf dem Siegel des Sadibbu (Abb. 15) dagegen steht der Wettergott in der (Mond-)Barke und hält in der rechten Hand das typische Blitzbündel, während er in der Linken ein

36 Radner 2002 (Anm. 10) 6-7.

${ }^{37}$ M. P. Streck, Nergal-ēreš, RlA 9 (1998-2001) 228. 
dem Stab auf unserem Siegel vergleichbares, mit Kugeln besetztes Zepter trägt. Auf dem besonders plastisch ausgearbeiteten, schon fast barock wirkenden Siegel des Aššur-šumu-iddina (Abb. 14) schließlich steht der Wettergott ${ }^{38}$ mit seiner Axt auf einem flügellosen Löwendrachen, dessen speiendes Feuer wie ein Blitzbündel stilisiert ist. Die Besonderheit dieses Siegels besteht in seiner Inschrift, die den Siegelinhaber als „Priester des Nergal (und) des Adad“39 identifiziert, deren Standarten auch abgebildet sind. Meines Erachtens belegt diese Inschrift, was wie Ikonographie ebenfalls deutlich macht: Eine saubere Trennung der Erscheinungsbilder der Gottheiten ist nicht (mehr) gegeben, die Aspekte von Nergal (Löwendrache) und Adad (Axt, Blitzbündel) mischen sich in synkretistischer Form ${ }^{40}$. Welcher Gott auf dem Siegel des Išme-ilu abgebildet ist, muss deshalb letztlich offen bleiben.

\section{Die Siegelung und die Siedlungsgeschichte von Dur-Katlimmu (Kühne)}

Die Bedeutung dieses Objektes für die Siedlungsgeschichte von DūrKatlimmu ist hochgradig. Erstmalig können die der Erdphase 9 zuzuordnenden Erdeinheiten und ihre zugehörige Architektur ${ }^{41}$, deren Erhaltungszustand sehr schlecht ist, durch einen absolut datierbaren Fund in stratifizierter Fundlage chronologisch fixiert werden. Die bisherige Aussage ${ }^{42}$, dass die Gründung der Unterstadt II wahrscheinlich im späten 8. vorchristlichen Jahrhundert erfolgt sei, muss zugunsten einer um hundert Jahre früheren Datierung revidiert werden. Der archäologische Befund deutet eine noch ältere Nutzung des Geländes an, die in die erste Hälfte des 9. oder sogar in das ausgehende 10. Jh. fallen könnte ${ }^{43}$.

38 U. Moortgat-Correns, Ein Kultbild Ninurtas aus neuassyrischer Zeit, Archiv für Orientforschung 35 (1988) 117-133, deutet dieses Bild dagegen als Darstellung des Gottes Ninurta. Vgl. auch Chikako E. Watanabe, Animal Symbolism in Mesopotamia (Wien 2002) 136-141.

39 Watanabe 1993 (Anm. 18) 112 Nr. 4.1.

40 D. Schwemer, Die Wettergottgestalten Mesopotamiens und Nordsyriens im Zeitalter der Keilschriftkulturen. Materialien und Studien nach den schriftlichen Quellen (Wiesbaden 2001) 613-625, nimmt zu dieser Problematik nicht direkt Stellung; indirekt geht die Vielfalt der Aspekte aber aus seinen Ausführungen hervor.

${ }^{41}$ Vgl. Anm. 17.

42 H. Kühne, Tall Šè Ḩamad - The Assyrian City of Dūr-Katlimmu: A Historic-Geographical Approach, in: T. Mikasa (Hg.), Essays on Ancient Anatolia in the Second Millennium B.C. (Wiesbaden 1998) 279. 287.

43 Ausführlich dazu der Bericht von M. Pucci (in Vorbereitung). 


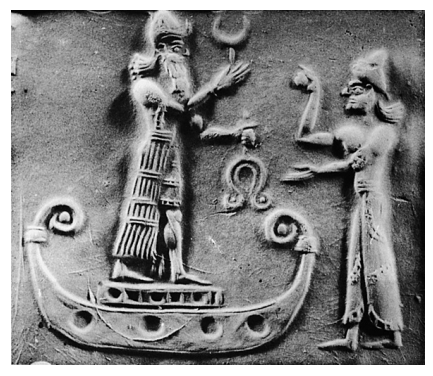

Abb. 17

Zur Zeit des Statthalters Nergal-ēreš (ca. 803-775) muss die Unterstadt II schon voll erschlossen und besiedelt gewesen sein. In den Inschriften der Stelen von Saba'a und Tall ar-Rimāḥ wird Dūr-Katlimmu als zugehörig zu seinem Regierungsbezirk Rașappa erwähnt, und es ist sehr erfreulich, nun den Bestätigungsnachweis von Dūr-Katlimmu selbst aus führen zu können. In der Inschrift der Stele von Tall ar-Rimāh ist Dūr-Katlimmu zudem deutlich in das Kolonisierungsprogramm des Königs Adad-nērārī III. eingebunden ${ }^{44}$. Dies kann als indirekter Beweis für die Besiedlung der Unterstadt II gewertet werden, denn Dūr-Katlimmu diente als zentraler Ort für die Bewirtschaftung und Verwaltung der neuen agronomischen Nutzungs- und Siedlungsgebiete in der Steppe und musste die notwendige Infrastruktur bereitstellen. Eine Kaufurkunde des Archivherrn Ibašši-ili aus dem ,Roten Haus ${ }^{45}$, die durch das Eponymat in das Jahr 828 datiert ist, hatte diese Datierung bislang schon nahegelegt, konnte aber nicht als Nachweis für eine Besiedlung der Unterstadt II gewertet werden. Sie fügt sich jetzt organischer in die Siedlungsgeschichte der Stadt ein.

44 Zusammenfassend und mit älterer Literatur: M. P. Streck, Nergal-ēreš, R1A 9 (1998-2001) 227-228.

45 Radner 2002 (Anm. 10) 157. 